\title{
Sub-Optimal Control Law for Minimum Energy Attitude Maneuver using CMGs
}

\author{
By Eijiro Uematsu ${ }^{1)}$, Seiya $\mathrm{UeNO}^{2)}$ and Takehiro $\mathrm{HIGUCHI}^{2)}$ \\ ${ }^{1)}$ Graduate School of Environment and Information Sciences, Yokohama National University, Yokohama, Japan \\ ${ }^{2)}$ Faculty of Environment and Information Sciences, Yokohama National University, Yokohama, Japan
}

(Received June 24th, 2013)

\begin{abstract}
Single Gimbal Control Moment Gyro (SGCMG) is an effective actuator for three axis attitude control of the satellites. Since satellite missions conduct with limited energy supplies in space, it is required to reduce the energy consumption during attitude maneuvers. However there are two problems with finding the optimal solutions for minimum energy maneuvers: the initial assumption and the calculation cost. To solve these problems, this study introduces a new sub-optimal control law to achieve the sub-minimum energy maneuvers without both initial assumption and iterative calculation. Compared to conventional steering law, the sub-optimal control law results in decreasing the energy consumption by more than 80 percent for all maneuver axis.
\end{abstract}

Key Words: Control Moment Gyro, Sub-Optimal Control Law, Minimum Energy Maneuvers

\section{Nomenclature}

$\boldsymbol{H}:$ : total angular momentum vector

$\omega$ : angular velocity vector of satellite

I : inertia matrix of satellite

h : CMG's angular momentum vector

$r$ : maneuver axis vector

$q \quad$ : quaternion vector

$\theta_{r} \quad: \quad$ maneuver angle

$h_{w}:$ angular momentum of flywheel

$\alpha_{i} \quad: \quad i$ th gimbal angle

$\beta \quad$ : pyramid skew angle

$A \quad$ : Jacobian matrix

$\boldsymbol{u}$ : gimbal angular velocity vector

$J \quad$ : criterion

$T_{\text {opt }}:$ maneuver time for optimal control

$\omega_{\mathrm{F} / \mathrm{F}}:$ angular velocity vector of sub-optimal feed-forward control law

$\boldsymbol{u}_{\mathrm{F} / \mathrm{F}}$ : gimbal angular velocity vector of suboptimal feed-forward control law

$\boldsymbol{u}_{0} \quad$ : initial gimbal angular velocity vector

$\boldsymbol{u}_{\mathrm{F} / \mathrm{B}}:$ gimbal angular velocity vector of feedback control law

$\tau_{r} \quad:$ required torque vector

$\boldsymbol{q}_{e}:$ quaternion error vector

$K_{p} \quad$ : proportional gain

$K_{d} \quad$ : derivative gain

$\lambda \quad$ : positive scalar

$\boldsymbol{E}_{3} \quad$ : unit matrix of order 3

$\boldsymbol{u}_{\text {com }}$ : gimbal angular velocity vector of combined control law

$t_{s} \quad$ : switching time of combined control law

$\boldsymbol{u}_{s u b}$ : gimbal angular velocity vector of suboptimal combined control law

$T_{\mathrm{f}} \quad:$ maneuver time

\section{Introduction}

Single Gimbal Control Moment Gyro (SGCMG) is a momentum exchange device which has better property of torque amplification than reaction wheels and double gimbal CMGs. Utilizing the SGCMG system will make the maneuver time shorter and energy consumption more efficient. In order to generate a desired torque, more than three SGCMG units are necessary for an attitude control system. Due to redundancy and complex form of the CMG system, a number of studies focus on control laws of a pyramid type 4-SGCMG system shown in Fig. 1. The biggest disadvantage of the pyramid type CMGs is a singular state where the system cannot produce output torque along a certain direction. To solve this problem, many singularity avoidance logics which allow torque error are developed. ${ }^{1-3)}$ Some researches conduct geometric analysis of singularities and clarify the passability of the singular surface. ${ }^{4,5}$ In addition, feed-forward control inputs are described by using the optimal control theory considering maneuver time, singularities, or energy consumption. $^{6-8)}$ Although optimal feed-forward control achieves singularity avoidance and optimal maneuvers, initial assumption and iterative calculations are needed to obtain optimal inputs. There are few studies about optimal control laws which have small calculation cost.

This study focuses on minimum energy maneuvers because satellite missions have to conduct with limited energy supplies in space. In order to overcome the optimal control problem mentioned above, this study introduces a new sub-optimal control law to calculate the input values without initial assumption and iterative calculations. The new control law is intended for an origin-symmetric satellite like a cube or a sphere. Based on the optimal solution of such a satellite, the sub-optimal control law will be designed. At first, the satellite 
angular velocity can be formulated as a quadratic function of time and the input value of each CMG unit as a linear function of time. Using the rotational equation of motion of the satellite with CMGs, the sub-optimal feed-forward control law is derived. Although the feed-forward control leads to the terminal attitude angle error, a feed-back control law adopted during the maneuver will modify the error. In this paper, the new control law composed of both the sub-optimal feed-forward control law and the feed-back control law will be presented. The objective of this study is to show the validity of the sub-optimal combined control law to achieve the sub-minimum energy maneuvers with low calculation cost.

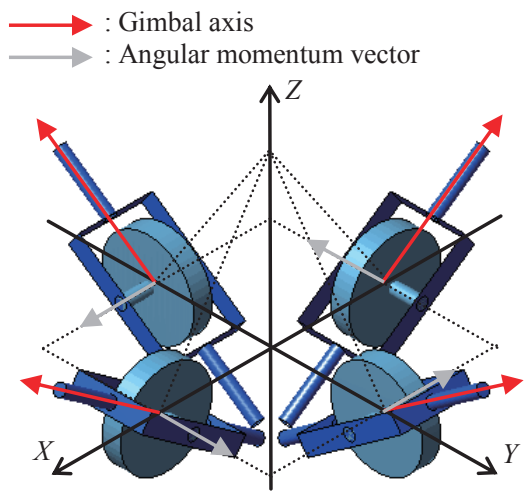

Fig. 1. Pyramid type 4-SGCMG system.

\section{Minimum Energy Maneuvers of Satellites}

This study considers sub-minimum energy maneuvers of a satellite. The sub-optimal control law is based on optimal solutions. In this chapter, the attitude parameterization, the equation of motion, and formulation of the energy minimization problem are described, followed by an example of calculation result of minimum energy maneuvers.

\subsection{Dynamics of satellites with CMGs}

The rotational equation of motion of a rigid satellite with CMGs, expressed by the body fixed coordinate, is given by

$$
\dot{\boldsymbol{H}}+\boldsymbol{\omega} \times \boldsymbol{H}=\boldsymbol{O}
$$

External torque is ignored during attitude maneuvers in this study. Total angular momentum vector $\boldsymbol{H}$ includes both the angular momentum of the satellite main body $I \boldsymbol{\omega}$ and the CMG system $\boldsymbol{h}$.

$$
\boldsymbol{H}=\boldsymbol{I} \boldsymbol{\omega}+\boldsymbol{h}
$$

Substituting Eq.(2) into Eq.(1) gives

$$
\boldsymbol{I} \dot{\boldsymbol{\omega}}=-\boldsymbol{\omega} \times \boldsymbol{I} \boldsymbol{\omega}-\boldsymbol{\omega} \times \boldsymbol{h}-\dot{\boldsymbol{h}}
$$

The attitude of the satellite is formulated by quaternions which consist of the maneuver axis $\boldsymbol{r}$ and the maneuver angle $\theta_{r}$.

$$
\begin{aligned}
\boldsymbol{r} & =\left[\begin{array}{lll}
l & m & n
\end{array}\right]^{\mathrm{T}} \\
\boldsymbol{q} & =\left[\begin{array}{llll}
q_{1} & q_{2} & q_{3} & q_{4}
\end{array}\right]^{\mathrm{T}} \\
& =\left[\begin{array}{llll}
l \sin \left(\frac{\theta_{r}}{2}\right) & m \sin \left(\frac{\theta_{r}}{2}\right) & n \sin \left(\frac{\theta_{r}}{2}\right) & \cos \left(\frac{\theta_{r}}{2}\right)
\end{array}\right]^{\mathrm{T}}
\end{aligned}
$$

The relation between the quaternion and the angular velocity of the satellite is expressed by

$$
\boldsymbol{\omega}=2\left[\begin{array}{cccc}
q_{4} & q_{3} & -q_{2} & -q_{1} \\
-q_{3} & q_{4} & q_{1} & -q_{2} \\
q_{2} & -q_{1} & q_{4} & -q_{3}
\end{array}\right]\left[\begin{array}{c}
\dot{q}_{1} \\
\dot{q}_{2} \\
\dot{q}_{3} \\
\dot{q}_{4}
\end{array}\right]
$$

The differential equation relating to the quaternion is derived from rearranging Eq.(6) as $\dot{\boldsymbol{q}}$.

$$
\dot{\boldsymbol{q}}=\frac{1}{2}\left[\begin{array}{cccc}
0 & \omega_{z} & -\omega_{y} & \omega_{x} \\
-\omega_{z} & 0 & \omega_{x} & \omega_{y} \\
\omega_{y} & -\omega_{x} & 0 & \omega_{z} \\
-\omega_{x} & -\omega_{y} & -\omega_{z} & 0
\end{array}\right]\left[\begin{array}{c}
q_{1} \\
q_{2} \\
q_{3} \\
q_{4}
\end{array}\right]
$$

A pyramid type 4-SGCMG system shown in Fig. 1 is considered as a simulation model in this study. All the gimbal angles are equal to 0 degrees in Fig. 1 and all the pyramid skew angles are the same value. The total angular momentum of pyramid type CMGs is expressed by sum of each CMG angular momentum vector as

$$
\begin{aligned}
\boldsymbol{h} & =\sum_{i=1}^{4} \boldsymbol{h}_{i} \\
& =h_{w}\left[\begin{array}{c}
-c \beta \sin \alpha_{1}-\cos \alpha_{2}+c \beta \sin \alpha_{3}+\cos \alpha_{4} \\
\cos \alpha_{1}-c \beta \sin \alpha_{2}-\cos \alpha_{3}+c \beta \sin \alpha_{4} \\
s \beta\left(\sin \alpha_{1}+\sin \alpha_{2}+\sin \alpha_{3}+\sin \alpha_{4}\right)
\end{array}\right]
\end{aligned}
$$

where $c \beta \equiv \cos \beta$ and $s \beta \equiv \sin \beta$. The time derivative of $\boldsymbol{h}$ is described by

$$
\dot{\boldsymbol{h}}=\boldsymbol{A} \boldsymbol{u}
$$

The Jacobian matrix $\boldsymbol{A}$ has dimension of $3 \times 4$ and the gimbal angular velocity vector $\boldsymbol{u}$ is the control input to the CMGs.

$$
\begin{gathered}
\boldsymbol{A}=h_{w}\left[\begin{array}{cccc}
-c \beta \cos \alpha_{1} & \sin \alpha_{2} & c \beta \cos \alpha_{3} & -\sin \alpha_{4} \\
-\sin \alpha_{1} & -c \beta \cos \alpha_{2} & \sin \alpha_{3} & c \beta \cos \alpha_{4} \\
s \beta \cos \alpha_{1} & s \beta \cos \alpha_{2} & s \beta \cos \alpha_{3} & s \beta \cos \alpha_{4}
\end{array}\right] \text { (10) } \\
\boldsymbol{u}=\dot{\boldsymbol{\alpha}}=\left[\begin{array}{llll}
\dot{\alpha}_{1} & \dot{\alpha}_{2} & \dot{\alpha}_{3} & \dot{\alpha}_{4}
\end{array}\right]^{\mathrm{T}}
\end{gathered}
$$

It is required to make the control input smaller for reduction of energy consumption during the maneuver. Based on the model mentioned above, the energy minimization problem is formulated in the following section.

\subsection{Formulation for minimum energy maneuver}

The satellite with the pyramid type CMGs has eleven state variables: angular velocities of the satellite, quaternions, and gimbal angles. The control input is each gimbal angular velocity shown in Eq.(11). To minimize the energy consumption, that is, power to drive the gimbal torque motor, the criterion is expressed by sum of squares of $\boldsymbol{u}$.

$$
J=\frac{1}{2} \int_{0}^{T_{\mathrm{opt}}}\left(\boldsymbol{u}^{\mathrm{T}} \boldsymbol{u}\right) d t
$$

The maneuver time for optimal control $T_{\text {opt }}$ is arbitrarily designated for numerical calculations.

This study takes into account a rest to rest maneuver for the simulations. The condition that the total CMG angular momentum equals to zero, therefore, is added to the terminal boundary condition.

$$
\boldsymbol{h}=\boldsymbol{0} \quad\left(t=T_{\text {opt }}\right)
$$

Moreover considering the periodic maneuvers of the satellite such as an earth observation satellite, the periodic 
boundary conditions that the terminal gimbal angles are equivalent to the initial gimbal angles are expressed.

$$
\alpha_{i}(0)=\alpha_{i}\left(T_{\text {opt }}\right)
$$

The additional initial condition of gimbal angles is required when starting the maneuver with designated initial gimbal angles. These energy minimization problems are treated as Two Point Boundary Value Problem (TPBVP) and solved with SCGRA (Sequential Conjugate Gradient Restoration Algorithm) in this study. ${ }^{9)}$

\subsection{Optimal solution}

In this section, the energy minimization problem is solved. For numerical calculations, parameters regarding the satellite with CMGs are set as Table 1. Note that the moment of inertia along each axis has same value. As shown in Fig. 2, an example of the minimum energy maneuver is 30 degrees rotation around the maneuver axis inclined with 40 degrees from $Z$ axis on $X Z$ plane. Fig. 3 illustrates the simulation result of the minimum energy maneuver.

Table 1. Simulation parameters.

\begin{tabular}{|c|c|}
\hline $\boldsymbol{I}$ & $\operatorname{diag}[100001000010000]\left(\mathrm{kgm}^{2}\right)$ \\
\hline$h_{w}$ & $1000\left(\mathrm{kgm}^{2} / \mathrm{s}\right)$ \\
\hline$\beta$ & $54.73(\mathrm{deg})$ \\
\hline$\alpha_{i}(0)$ & $0(\mathrm{deg})$ \\
\hline$T_{\mathrm{opt}}$ & $30(\mathrm{~s})$ \\
\hline $\boldsymbol{r}$ & {$\left[\sin \left(\frac{2}{9} \pi\right) 0 \quad \cos \left(\frac{2}{9} \pi\right)\right]^{\mathrm{T}}$} \\
\hline$\theta_{r}$ & $30(\operatorname{deg})$ \\
\hline
\end{tabular}

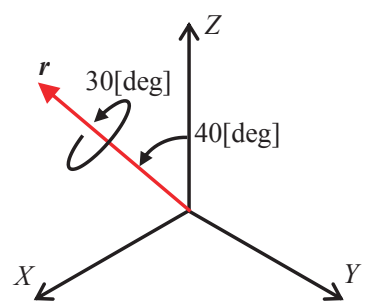

Fig. 2. Example of maneuver axis.

As shown in Fig. 3(b), minimizing energy consumption leads to the input value which can be regarded as a linear function of time. Moreover, the optimal inputs are roughly symmetrical about the middle point of maneuver, 15 seconds in this case, to satisfy the boundary condition shown in Eq.(14). Similarly, Fig. 3(a) indicates the angular velocity of the satellite is symmetrical and similar to a quadratic function of time. On the basis of these characteristics of optimal solutions mentioned above, formulation of the feed-forward control law for sub-minimum energy maneuvers will be described in the following chapter.

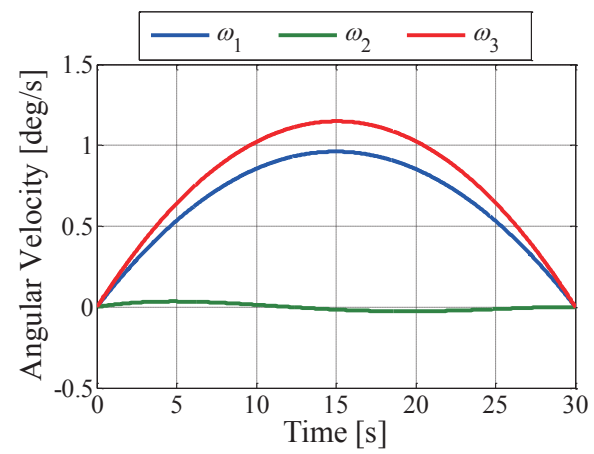

(a) Angular velocity of satellite

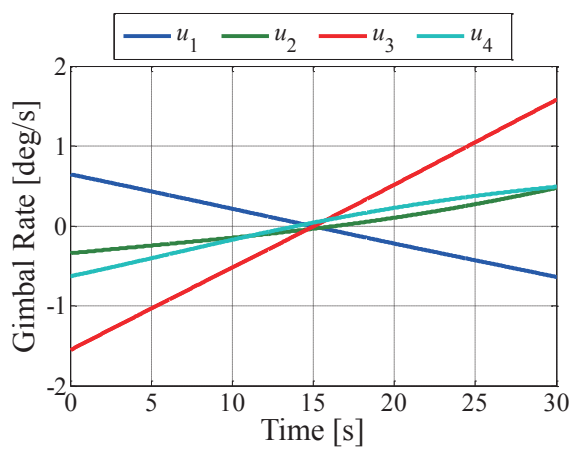

(b) Gimbal angular velocity

Fig. 3. Simulation result of optimal control.

\section{Sub-Optimal Feed-Forward Control Law}

In this chapter, sub-optimal feed-forward control law is designed. The control law can calculate sub-optimal input values from required attitude directly. Compared to the optimal solution, the validity of the feed-forward control law will be proven.

\subsection{Formulation of sub-optimal control law}

The optimal input and the angular velocity of satellite can be approximated by a linear function and a quadratic function of time, respectively. First, the symmetrical angular velocity of the satellite is formulated as

$$
\omega=\omega_{a}\left(t-\frac{T_{\mathrm{opt}}}{2}\right)^{2}-\frac{T_{\mathrm{opt}}^{2}}{4} \omega_{a}
$$

where $\omega_{a}$ is unknown coefficient vector. The integral of the angular velocity gives the maneuver angle.

$$
\int_{0}^{T_{\text {opt }}} \boldsymbol{\omega} d t=\theta_{r} \boldsymbol{r}
$$

Substituting Eq.(15) into Eq.(16) gives

$$
\omega_{a}=-\frac{6 \theta_{r}}{T_{\mathrm{opt}}^{3}} \boldsymbol{r}
$$

Therefore, the angular velocity as the sub-optimal solution is derived.

$$
\omega_{\mathrm{F} / \mathrm{F}}=\left\{-\frac{6 \theta_{r}}{T_{\mathrm{opt}}^{3}}\left(t-\frac{T_{\mathrm{opt}}}{2}\right)^{2}+\frac{3 \theta_{r}}{2 T_{\mathrm{opt}}}\right\} \boldsymbol{r}
$$

Second, the gimbal angular velocity can be formulated as the linear function of time.

$$
\boldsymbol{u}_{\mathrm{F} / \mathrm{F}}=\left(-\frac{2}{T_{\mathrm{opt}}} t+1\right) \boldsymbol{u}_{0}
$$


Eq.(3) and Eq.(9) are used in order to clarify the initial gimbal angular velocity $\boldsymbol{u}_{0}$. At the initial time of maneuver, the following equation is derived.

$$
\boldsymbol{I} \dot{\boldsymbol{\omega}}(0)+h_{w} \boldsymbol{A}(0) \boldsymbol{u}_{0}=0
$$

The time derivative of the angular velocity of satellite is described by

$$
\dot{\omega}_{\mathrm{F} / \mathrm{F}}=-\frac{12 \theta_{r}}{T_{\mathrm{opt}}^{3}}\left(t-\frac{T_{\mathrm{opt}}}{2}\right) \boldsymbol{r}
$$

Substituting $\quad \dot{\omega}_{\mathrm{F} / \mathrm{F}}(0)=\frac{6 \theta_{r}}{T_{\mathrm{opt}}^{2}} \boldsymbol{r}$ and $A(0) \equiv A_{0} \quad$ into Eq. (20) leads to

$$
\boldsymbol{A}_{0} \boldsymbol{u}_{0}=-\frac{6 \theta_{r}}{h_{w} T_{\mathrm{opt}}^{2}} \boldsymbol{I r}=\boldsymbol{W}
$$

Thus, the initial gimbal angular velocity is obtained by using the pseudo inverse matrix.

$$
\boldsymbol{u}_{0}=\boldsymbol{A}_{0}^{\mathrm{T}}\left(\boldsymbol{A}_{0} \boldsymbol{A}_{0}^{\mathrm{T}}\right)^{-1} \boldsymbol{W}
$$

The relationship between Eq.(23) and energy consumption is described as follows. The pseudo inverse matrix makes the norm of initial gimbal angular velocity minimum. From Eq.(12) and Eq.(19), the integrand of the criterion $L$ can be expressed as the function of the norm.

$$
\begin{aligned}
L & =\frac{1}{2}\left(u_{\mathrm{F} / \mathrm{F} 1}^{2}+u_{\mathrm{F} / \mathrm{F} 2}^{2}+u_{\mathrm{F} / \mathrm{F} 3}^{2}+u_{\mathrm{F} / \mathrm{F} 4}^{2}\right) \\
& =\frac{1}{2}\left(-\frac{2}{T_{\mathrm{opt}}} t+1\right)^{2}\left\|\boldsymbol{u}_{0}\right\|
\end{aligned}
$$

Eq.(24) shows $\boldsymbol{u}_{0}$ as the function of $\boldsymbol{u}_{\mathrm{F} / \mathrm{F}}$ minimizes the criterion. As a result, the sub-optimal input value is derived.

$$
\boldsymbol{u}_{\mathrm{F} / \mathrm{F}}=\left(-\frac{2}{T_{\text {opt }}} t+1\right) \boldsymbol{A}_{0}^{\mathrm{T}}\left(\boldsymbol{A}_{0} \boldsymbol{A}_{0}^{\mathrm{T}}\right)^{-1} \boldsymbol{W}
$$

This new control law realizes both sub-minimum energy maneuvers and low calculation cost enough to obtain the sub-optimal input from the required attitude directly. Although there is a possibility that the CMG system falls into singularities due to the pseudo inverse matrix, it is easy to avoid the singularities. As shown in Eq.(25), the singular state may occur not during the maneuver but at the initial time of maneuver. Changing the combination of initial gimbal angles by null motion before starting the maneuver enables the satellite to conduct the maneuver without the singularities.

\subsection{Validity of sub-optimal control law}

Making sure the validity of the sub-optimal control law, numerical calculations with the simulation conditions described in Table 1 and Fig. 2 are conducted. The simulation results of the sub-optimal control law are illustrated in Fig. 4. Table 2 shows the criterion and the terminal attitude angle error in the case of maneuver using both the sub-optimal control law and the optimal control theory. Table 2 indicates that although the sub-optimal control law leads to 1.62 degrees terminal error, the increment of criterion is less than 4 percent than that of optimal calculation. In addition, the new control law does not require the initial assumption and the iterative calculation which are needed for the numerical calculation using the optimal control theory. Thus, the sub-optimal control law achieves the sub-minimum energy maneuver with low calculation costs.

The reason why the terminal error occurs is that the sub-optimal input is derived considering the initial condition as shown in Eq.(20). So, the angular velocity as the sub-optimal solution formulated in Eq.(18) is accurately reflected only at the initial time. During the maneuver conducted above, for example, there should be no angular velocity about $Y$ axis because the maneuver axis has no $Y$ component. As shown in Fig. 4(a), while satisfying the initial time condition, that is $\left(\dot{\omega}_{\mathrm{F} / \mathrm{F}}\right)_{y}=0$, there are some errors except the initial time. The attitude error which occurs during the maneuver remains until the terminal state of maneuver. Hence, the sub-optimal control law results in the terminal attitude angle error. In order to overcome this problem, a feed-back control is added to the sub-optimal feed-forward control law in the following chapter.

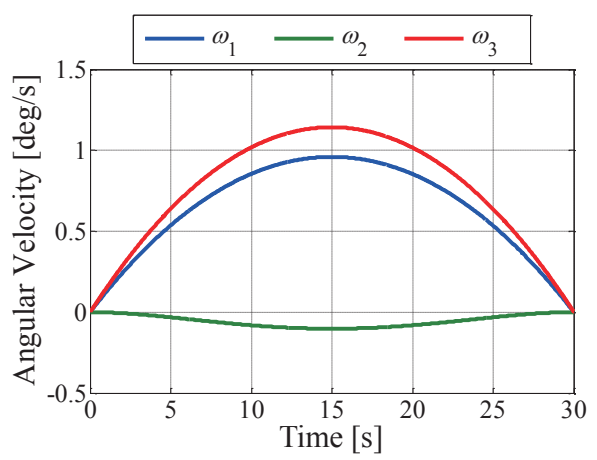

(a) Angular velocity of satellite

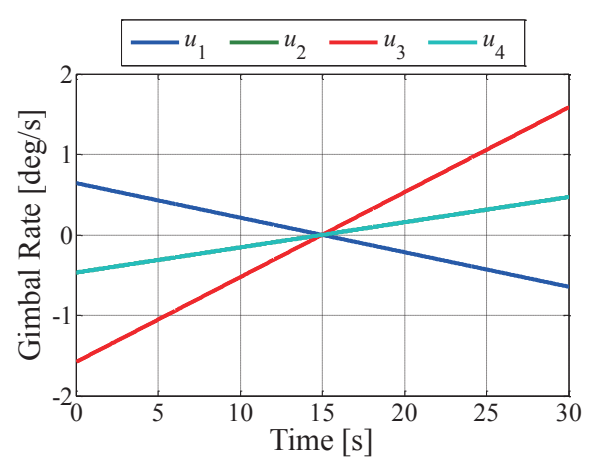

(b) Gimbal angular velocity

Fig. 4. Simulation result of sub-optimal control.

Table 2. Validity of sub-optimal control law.

\begin{tabular}{|c|c|c|}
\hline & Sub-optimal & Optimal \\
\hline Criterion $J[1 / \mathrm{s}]$ & 0.00512 & 0.00494 \\
\hline Terminal error $[\mathrm{deg}]$ & 1.62 & ㄴ.
\end{tabular}

\section{Sub-Optimal Combined Control Law}

The sub-optimal feed-forward control law mentioned in the previous chapter triggers the attitude angle error during maneuvers. To modify the error, the feed-back control law is presented. Moreover, the combined control law which has merits of both feed-forward control and feed-back control will be proposed.

\subsection{Feed-back control law}

The pseudo inverse steering law is utilized as the feed-back control law in this study. Based on the inverse kinematics, the 
control input can be derived from Eq.(9).

$$
\boldsymbol{u}_{\mathrm{F} / \mathrm{B}}=\boldsymbol{A}^{\mathrm{T}}\left(\boldsymbol{A} \boldsymbol{A}^{\mathrm{T}}\right)^{-1}\left(-\boldsymbol{\tau}_{r}-\boldsymbol{\omega} \times \boldsymbol{h}\right)
$$

The required torque $\boldsymbol{\tau}_{r}$ is composed of the quaternion error $\boldsymbol{q}_{e}$.

$$
\boldsymbol{\tau}_{r}=-K_{p} \boldsymbol{q}_{e}-K_{d} \dot{\boldsymbol{q}}_{e}
$$

The problem that the pseudo inverse steering law may cause the singular state of CMG system, can be solved by utilizing SR-inverse method, which attempts avoidance by allowing torque errors. The SR-inverse method is easy to apply to this control law because only $\lambda \boldsymbol{E}_{3}$ can be added in Eq.(26).

$$
\boldsymbol{u}_{\mathrm{F} / \mathrm{B}}=\boldsymbol{A}^{\mathrm{T}}\left(\boldsymbol{A} \boldsymbol{A}^{\mathrm{T}}+\lambda \boldsymbol{E}_{3}\right)^{-1}\left(-\boldsymbol{\tau}_{r}-\boldsymbol{\omega} \times \boldsymbol{h}\right)
$$

However, there is very little possibility of falling into singularities during 30 degrees rotation on the rest-to-rest maneuver with low energy consumption. This paper considers no singular state during maneuvers and will use the pseudo inverse steering law shown in Eq.(26) for the numerical calculations.

\subsection{Combined control law}

There are two types of combined control laws. One is the control law which switches from the sub-optimal feed-forward control law to the feed-back control law. Although this combined control law is simple to be adapted, there is a discontinuous change at the switching time. In the case of the simulation conditions shown in Table 1 and Fig. 2, the combined control whose switching time is equal to 15 seconds is illustrated in Fig. 5.

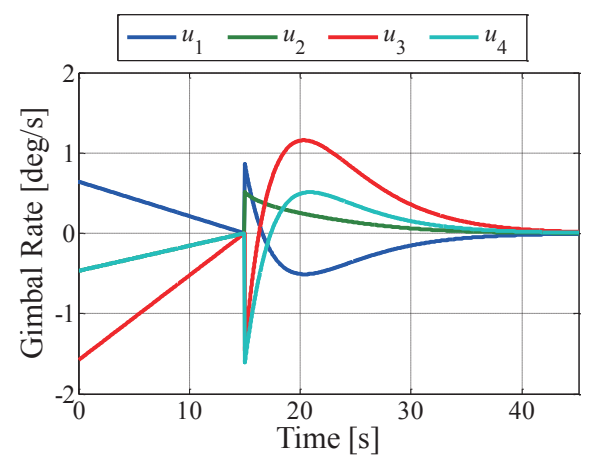

Fig. 5. Switching control law.

As shown in Fig. 5, the discrete input which occurs at 15 seconds puts an extra load on the CMG unit and may result in breakdown of the CMG system.

The other control law uses the feed-forward and the feed-back control law simultaneously. In this control law, the satellite starts maneuvering only with the sub-optimal feed-forward control law at first. Then, both will work from the switching time $t_{s}$ which can be designated arbitrarily. As the time passes, the percentage of feed-back input increases in inverse proportion to the feed-forward input. Eq.(29) describes the combined control input $\boldsymbol{u}_{\text {com }}$.

$$
\boldsymbol{u}_{c o m}=\frac{T_{\mathrm{opt}}-t}{T_{\mathrm{opt}}-t_{s}} \boldsymbol{u}_{\mathrm{F} / \mathrm{F}}+\frac{t-t_{s}}{T_{\mathrm{opt}}-t_{s}} \boldsymbol{u}_{\mathrm{F} / \mathrm{B}}
$$

Finally, the only feed-back control is applied to modify the terminal attitude error.

Consequently, the new sub-optimal control law is composed of three phases: feed-forward control, combined control, and feed-back control. The sub-optimal combined control input $\boldsymbol{u}_{s u b}$ can be described as follows.

$$
\boldsymbol{u}_{s u b}= \begin{cases}\boldsymbol{u}_{\mathrm{F} / \mathrm{F}} & \left(0<t<t_{s}\right) \\ \boldsymbol{u}_{c o m} & \left(t_{s} \leq t<T_{\mathrm{opt}}\right) \\ \boldsymbol{u}_{\mathrm{F} / \mathrm{B}} & \left(T_{\mathrm{opt}} \leq t\right)\end{cases}
$$

\section{Simulation Results}

In this chapter, the new sub-optimal combined control law mentioned in the previous chapter will be demonstrated. The simulation result of the new control law is compared with that of the conventional pseudo inverse steering law. Moreover, the effect of maneuver axis on the energy consumption is clarified.

\subsection{Simulation conditions}

Table 1 and Fig. 2 are considered as the simulation parameters. In addition, the derivative gain $K_{d}$, the switching time $t_{s}$, the maneuver time for sub-optimal input $T_{\mathrm{opt}}$, and the settling time $T_{\mathrm{f}}$ are shown in Table 3. As shown in Eq.(31), the terminal time of criterion is set to the settling time.

10,15 , and 20 seconds are applied as the switching time $t_{s}$ which shifts $\boldsymbol{u}_{\mathrm{F} / \mathrm{F}}$ to $\boldsymbol{u}_{\text {com }}$ as expressed in Eq.(30). It is expected that using the half of $T_{\text {opt }}$ as the switching time will decrease the criterion. The faster switching time will not bring the merit of feed-forward control, whereas the slower switching time will lead to the large attitude error. For this reason, three conditions of the switching time are compared to investigate the effect of $t_{s}$ on the criterion.

The new control law has three phases which are switched by $t_{s}$ and $T_{\text {opt }}$. The settling time $T_{f}$ is chosen as 45 seconds in order for each phase time to be roughly the same value. Rapid maneuver will be achieved when the shorter settling time is employed. In the other hand, the percentage of feed-back control will increase which causes large discrete input at the switching time and the increment of the criterion. The proportional gain $K_{p}$ is adjusted so as to fix the settling time.

Table 3. Simulation parameters for combined control law.

\begin{tabular}{|c|c|c|c|}
\hline$K_{d}$ & \multicolumn{3}{|c|}{10000} \\
\hline$t_{s}$ & $10(\mathrm{~s})$ & $15(\mathrm{~s})$ & $20(\mathrm{~s})$ \\
\hline$T_{\mathrm{opt}}$ & \multicolumn{3}{|c|}{$30(\mathrm{~s})$} \\
\hline$T_{\mathrm{f}}$ & \multicolumn{3}{|c|}{ Around 45 (s) } \\
\hline
\end{tabular}

$$
J=\frac{1}{2} \int_{0}^{T_{\mathrm{f}}}\left(\boldsymbol{u}^{\mathrm{T}} \boldsymbol{u}\right) d t
$$

\subsection{Simulation results}

As an example, the simulation result of the sub-optimal combined control law whose switching time is equivalent to 15 seconds is illustrated in Fig. 6. Similarly, the simulation result obtained by the only pseudo inverse steering law is described in Fig. 7. As a result, Table 4 shows the criterion in the case of maneuver using the sub-optimal control law and the pseudo inverse steering law. 


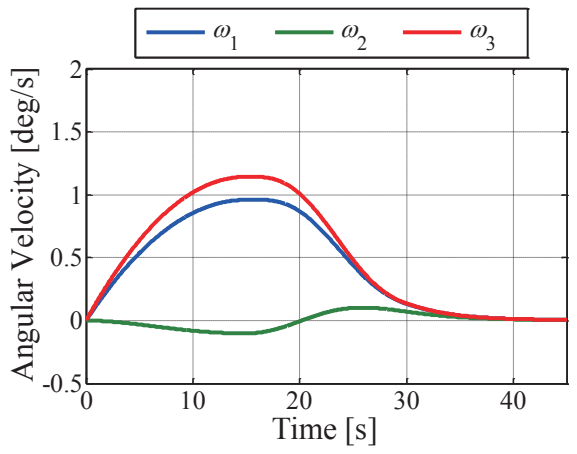

(a) Angular velocity of satellite

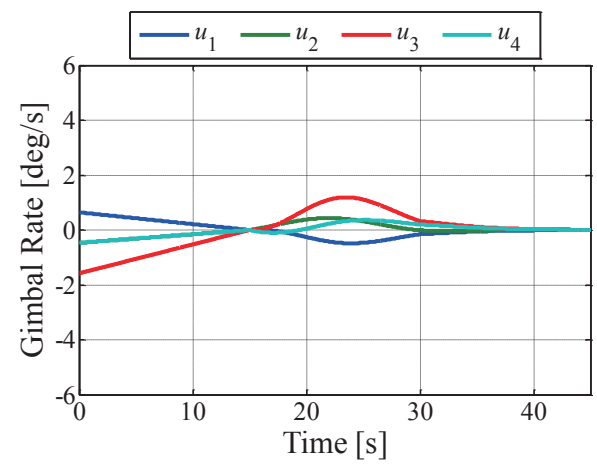

(b) Gimbal angular velocity

Fig. 6. Simulation result of sub-optimal control $\left(t_{s}=15[\mathrm{~s}]\right)$.

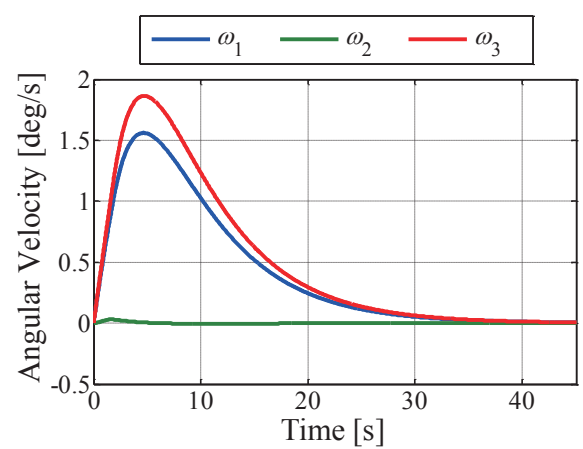

(a) Angular velocity of satellite

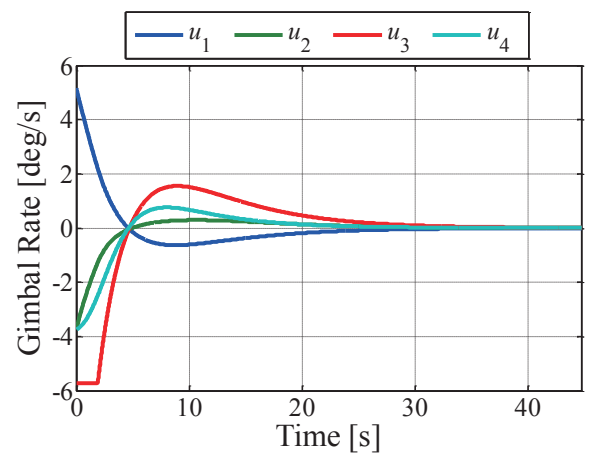

(b) Gimbal angular velocity

Fig. 7. Simulation result of pseudo inverse steering law.
Table 4. Comparison of criterion.

\begin{tabular}{|c|c|}
\hline & Criterion $J[1 / \mathrm{s}]$ \\
\hline Sub-optimal $\left(t_{s}=10\right)$ & 0.00537 \\
\hline Sub-optimal $\left(t_{s}=15\right)$ & 0.00461 \\
\hline Sub-optimal $\left(t_{s}=20\right)$ & 0.00464 \\
\hline Pseudo inverse steering & 0.0273 \\
\hline
\end{tabular}

Table 4 indicates that the switching time has an impact on the criterion. In particular, the switching time set to 15 seconds reduces the criterion because the time as 15 seconds is the middle point of the maneuver. Since the gimbal angular velocities in the sub-optimal feed-forward control law are zero in 15 seconds, it is easy to switch to the other control law at the midpoint of the maneuver time. Though there may be the switching time minimizing the criterion other than 15 seconds, the change rate of criterion between the switching time set to 15 seconds and 20 seconds is less than 1 percent. Therefore, it should be emphasized that the switching time of 15 seconds is appropriate to the sub-minimum energy maneuvers.

Compared to the pseudo inverse steering law, the criterion of sub-optimal control law set to 15 seconds as the switching time is reduced by 83 percent. Although the pseudo inverse steering law employs the local minimum input, the large input is needed to approach the required attitude at the initial time. In conclusion, the sub-optimal combined control law realizes both the sub-minimum energy maneuvers and low calculation cost.

\subsection{Effect of maneuver axis}

Simulation results described in the previous section regard the rotation around only one maneuver axis shown in Fig. 2. In order to verify the applicability of the sub-optimal control law to all maneuver axis, the effect of maneuver axis is clarified. The definition of maneuver axis is illustrated in Fig 8 and three cases of maneuver axis are considered: $X Z$ plane, $X Y$ plane, and $X Y Z$ space. Table 5 shows the maneuver conditions. Although $\theta_{1}$ and $\theta_{2}$ range from 0 to 90 degrees, it is expected to obtain the same results for ranging from 0 to 360 degrees because of the symmetry of the pyramid type CMG system. Fig. 9 describes the criterion in the case of using the sub-optimal control law and the conventional pseudo inverse steering law.

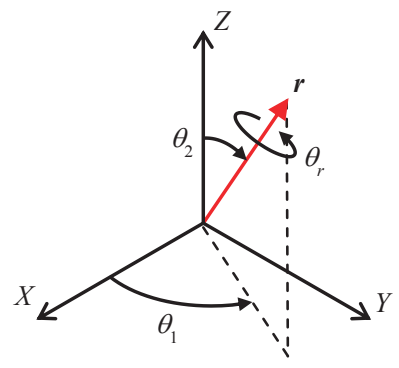

Fig. 8. Definition of maneuver axis. 
Table 5. Maneuver conditions.

\begin{tabular}{|c|c|c|}
\hline & $\theta_{1}[\mathrm{deg}]$ & $\theta_{2}[\mathrm{deg}]$ \\
\hline$X Z$ plane & 0 & $0 \sim 90$ \\
\hline$X Y$ plane & $0 \sim 90$ & 90 \\
\hline \multirow{2}{*}{$X Y Z$ space } & $0 \sim 90$ & 45 \\
\cline { 2 - 3 } & 45 & $0 \sim 90$ \\
\hline
\end{tabular}

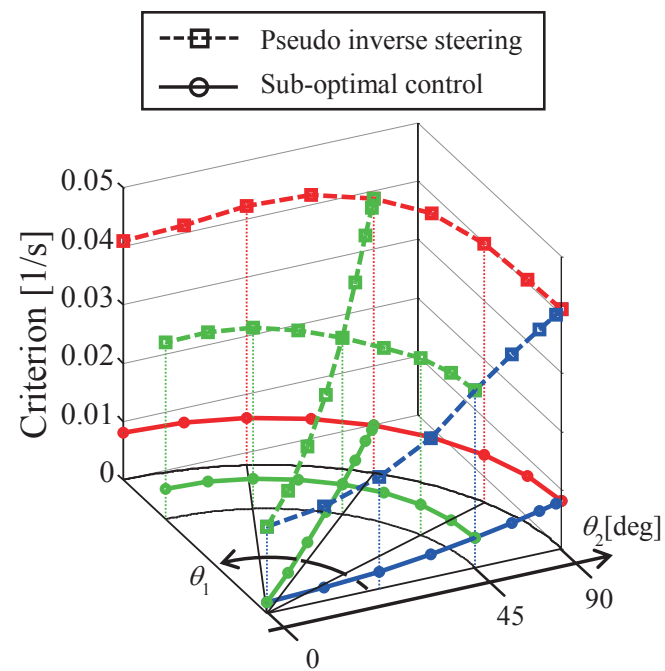

Fig. 9. Criterion for all maneuver axis.

Fig. 9 indicates the high energy performance of the sub-optimal control law. When the maneuver axis lies on $X Y$ plane illustrated by the red line, for example, the average value of the criterion using the pseudo inverse steering law equals to $0.0438[1 / \mathrm{s}]$. By contrast, the average using the sub-optimal control law is equivalent to $0.00812[1 / \mathrm{s}]$. Therefore, 81 percent decrements of the criterion are achieved on $X Y$ plane on average. Similarly, 82 percent and 83 percent decrements are completed on $X Z$ plane showed by blue line and in $X Y Z$ space by green line, respectively. In $X Y Z$ space, calculation results using other angles of $\theta_{1}$ and $\theta_{2}$ denote the same tendency of green line. As a consequence, the new sub-optimal control law achieves reduction of the energy consumption for all maneuver axis. It is verified that compared to the pseudo inverse steering law, the criterion is reduced by more than 80 percent in all maneuvers.

\section{Conclusions}

The sub-optimal combined control law is designed on the basis of optimal solutions of the origin-symmetric satellite. The new control law consists of three phases: the sub-optimal feed-forward control phase, the combined control phase, and the feed-back control phase. The sub-optimal feed-forward control law results in the sub-minimum energy maneuver where the difference with the optimal solution is less than 5 percent. In addition, the control law can be adapted to all maneuver axis because it can be derived analytically. The terminal attitude angle error caused by the feed-forward control law is modified by employing the pseudo inverse steering law as the feed-back control law.

In the combined control phase, the term of feed-back input works gradually so as not to cause a discontinuous input at the switching time from the sub-optimal feed-forward control phase to the combined control phase. Therefore, this control law does not put an extra load on the CMG system and is useful for actual attitude maneuvers. As a result of the control law, energy consumption is reduced by more than 80 percent for all maneuver axis in comparison with the case with only pseudo inverse steering law. It is emphasized that the new sub-optimal control law has a great benefit with respect to both optimality and calculation cost.

\section{References}

1) Nakamura, Y. and Hanafusa, H.: Inverse Kinematic Solutions with Singularity Robustness for Robot Manipulator Control, Journal of Dynamic Systems, Measurement, and Control, 108(1986), pp. 163-171.

2) Ford, K. A. and Hall, C. D. : Singular Direction Avoidance Steering for Control-Moment Gyros, Journal of Guidance, Control, and Dynamics, 23(2000), pp. 648-656.

3) Wie, B., Bailey, D., and Heiberg, C.: Singularity Robust Steering Logic for Redundant Single-Gimbal Control Moment Gyros, Journal of Guidance, Control, and Dynamics, 24(2001), pp. 865-872.

4) Kurokawa, H.: A Geometric Study of Single Gimbal Control Moment Gyros - Singularity Problems and Steering Law -, Report of Mechanical Engineering Laboratory, No. 175 (1998).

5) Wie, B.: Singularity Analysis and Visualization for Singule-Gimbal Control Moment Gyro System, Journal of Guidance, Control, and Dynamics, 27(2004), pp. 271-281.

6) Hoelscher, B. R. and Vadali, S. R.: Optimal Open-Loop and Feedback Control Using Single Gimbal Control Moment Gyroscopes, Journal of the Astronautical Sciences, 42(1994), pp. 189-206.

7) Vadali, S. R. and Krishnan, S.: Suboptimal Command Generation for Control Moment Gyroscopes and Feedback Control of Spacecraft, Journal of Guidance, Control, and Dynamics, 18(1995), pp. 1350-1354.

8) Akiyama, K., Fujihashi, K. and Matsunaga, S.: High-Speed Attitude Control System for Small Satellite with Micro-CMGs, Transactions of JSASS, Aerospace Technology Japan, 8(2010), pp. Pd_91-Pd_97.

9) $\mathrm{Wu}$, A. K. and Miele, A.: Sequential Conjugate GradientRestoration Algorithm for Optimal Control Problems with Non-Differential Constraints and General Boundary Conditions, Part1, Optimal Control Applications \& Methods, 1 (1980). 